\title{
Cloning, tissue distribution, expression pattern, and function of porcine maternal embryonic leucine zipper kinase
}

\author{
Pengyuan Chen ${ }^{1 \#}$, Jiaqiang Wang ${ }^{2 *}$, Xingye Wang ${ }^{3}$, Xiaolin Chen ${ }^{3}$, Chunling $\mathrm{Li}^{1}$, Taichang $\operatorname{Tan}^{2}$ \\ ${ }^{1}$ Sichuan Academy of Medical Sciences \& Sichuan Provincial People's Hospital, Chengdu 610072, China; ${ }^{2}$ Department of Laboratory Medicine, \\ Sichuan Provincial People's Hospital, University of Electronic Science and Technology of China, Chengdu 611731, China; ${ }^{3}$ College of Veterinary \\ Medicine, Northwest A\&F University, Yangling 712100, China \\ Contributions: (I) Conception and design: P Chen, J Wang; (II) Administrative support: C Li, T Tan; (III) Provision of study materials: X Wang; (IV) \\ Collection and assembly of data: J Wang, X Chen; (V) Data analysis and interpretation: P Chen, J wang; (VI) Manuscript writing: All authors; (VII) \\ Final approval of manuscript: All authors. \\ \#These authors contributed equally to this work. \\ Correspondence to: Chunling Li. Sichuan Provincial People's Hospital, University of Electronic Science and Technology of China, Chengdu 610072, \\ China. Email: 1c1206778081@163.com; Taichang Tan. Department of Laboratory Medicine, Sichuan Provincial People’s Hospital, University of \\ Electronic Science and Technology of China, Chengdu 611731, China. Email: sinotan@sina.com.
}

Background: Maternal embryonic leucine zipper kinase (MELK) is an atypical member of the snf1/AMPK family of serine-threonine kinases, involved in diverse physiological and pathological processes, including cell proliferation, apoptosis, embryogenesis, cancer treatment resistance, and RNA processing. MELK is highly expressed in human cancers and is associated with more aggressive forms of astrocytoma, glioblastoma, breast cancer, and melanoma to date, no information about porcine MELK (pMELK) has been reported.

Methods: In this study, the pMELK coding sequence was cloned from swine spleen and characterized. We also quantitatively determined the expression of MELK in 11 tissues isolated from a piglet and determined its subcellular localization when expressed in swine umbilical vein endothelial cells (SUVEC) as a fusion protein. Moreover, we report the functional characterization of pMELK protein concerning its role in apoptosis.

Results: Sequencing analysis showed that full-length of pMELK is 2,072 bp with 17 exons, encoding 655 amino acids, including an S-TKc conserved domain. Comparison of pMELK with ten other mammalian species of their orthologous sequences showed $>91 \%$ homology and an evolutionary distance $<0.05$, demonstrating that MELK is highly conserved in evolution. Relative quantification of MELK expression in 11 tissue samples isolated from 30-day-old piglets showed MELK expression in all tested organs and the highest expression in the superficial inguinal lymph node. Constructed a plasmid named pEGFP-MELK, and the fusion protein GFP-MELK was successfully expressed in SUVECs. Fluorescence microscopy revealed the subcellular distribution of the fusion protein GFP-MELK was limited to the cytoplasm. About function, Flow cytometry analysis showed that overexpression of GFP-pMELK in SUVEC cells enhances staurosporine (STS)—induced apoptosis, but not significantly different. The pMELK protein also was found to interact with porcine BCL-G and transient transfection of the recombinant plasmid pCMV-HApMELK into SUVEC cells stably expressing GFP-pBCL-G protein inhibited pBCL-G -induced apoptosis significantly.

Conclusions: The present study provided useful information on pMELK basic details and function in apoptosis offer a potential new molecular model for disease interventions and disease related to human MELK and BCL-G.

Keywords: Porcine MELK ( $\mathrm{p} M E L K)$; cloning; tissue distribution; expression pattern; function

Submitted Jan 18, 2020. Accepted for publication Feb 24, 2020.

doi: $10.21037 / \mathrm{atm} .2020 .03 .46$

View this article at: http://dx.doi.org/10.21037/atm.2020.03.46 


\section{Introduction}

Maternal embryonic leucine zipper kinase $(M E L K)$ is a member of the Snf1/AMPK family of kinases (1-3), which participates in a wide range of cellular processes related to the cell cycle $(4,5)$, apoptosis, spliceosome assembly (6), and cell proliferation (7). MELK is highly expressed in human cancers and is associated with more aggressive forms of astrocytoma, glioblastoma, breast cancer, and melanoma $(8,9)$, suggesting that $M E L K$ is a potential anticancer target in diverse tumor entities (10). According to the studies of oncogenic signal transduction pathways, MELK plays a vital role in the regulation of signal transduction and complex coordination, particularly in pathways relating to cancer cell growth and signaling $(11,12)$. Besides, MELK can covalently attach to various proteins, including MAPK, p53, FOXM1, c-JUN, and BCL-G (a pro-apoptotic member of the BCL-2 family), affect the properties of cancer stem cells (10).

The effects of MELK in apoptosis are controversial, since both promotes and inhibits apoptosis is existing. For example, in mice, the physical association between MELK and apoptosis signal-regulating kinase 1 (ASK1) stimulated $\mathrm{H}_{2} \mathrm{O}_{2}$-mediated apoptosis by enhancing ASK1 activity in embryonic kidney and hematopoietic cells (13). In HCT116 colon cancer cells, there is a positive correlation between MELK and $\mathrm{p} 53$ expression, and overexpression of MELK increases $\mathrm{p} 53$ expression (14). This data supplies evidence that MELK may have a vital role in promoting apoptosis in some kinds of cancer. However, data from other reports support the opposite conclusion. In glioblastoma (a highly malignant brain cancer) cells, gene silencing of MELK resulted in increased $\mathrm{p} 53$ expression and consequent p53-dependent apoptosis (15). Lin et al. also confirmed that down-regulation of $M E L K$ expression using siRNA markedly inhibits breast cancer cell growth (16). Moreover, MELK has an ambiguous role in promoting both cell division and cell death in Caenorbabditis elegans $(17,18)$. Overall, MELK may play dual roles in different cancers, and further study is required to determine the pro-apoptotic and anti-apoptotic biological functions of MELK accurately.

In this study, the porcine $M E L K$ ( $\mathrm{p} M E L K)$ coding sequence was cloned from swine spleen and characterized. We also quantitatively determined the expression of $M E L K$ in 11 tissues isolated from a piglet and determined its subcellular localization when expressed in swine umbilical vein endothelial cells (SUVEC) as a fusion protein. Moreover, we report the functional characterization of pMELK protein concerning its role in apoptosis. So, the study provides useful information on pMELK basic details and function in apoptosis, offers a potential new molecular model for disease interventions and disease related to human MELK.

\section{Methods}

\section{Swine}

Three 30-day-old healthy Landrace piglets were bought from Youhai piglet farm (Shaanxi, China). All animal experiments were conducted in compliance with current Chinese ethical legislation.

\section{Cell lines and reagents}

Prof. Yan-Ming Zhang kindly offered SUVEC, College of Veterinary Medicine, Northwest A\&F University (19), was cultured in $10 \%$ fetal bovine serum (FBS; Gibco) at $5 \% \mathrm{CO}_{2}, 37{ }^{\circ} \mathrm{C}$. Staurosporine (STS) was purchased from Sigma-Aldrich (MO, USA). HA-tag and MYCtag monoclonal antibodies were bought from Santa Cruz Biotechnology, Inc. (Santa Cruz, CA, USA). Anti-mouse horseradish peroxidase (HRP)-conjugated antibody was obtained from Sigma-Aldrich.

\section{$R N A$ extraction and real-time $q P C R$ analysis}

Heart, liver, spleen, lung, kidney, tonsil, thymus, superficial inguinal lymph node, hilar lymph node, mesenteric lymph node, and chin lymph node were isolated from the piglets and marked seriously. Washed the tissues with Physiological saline three times, and $100 \mathrm{mg}$ of each origin were homogenized and diluted 1:10 with phosphate-buffered saline (PBS; $0.1 \mathrm{M}, \mathrm{pH}$ 7.4). Total RNA was isolated from the homogenized using TRIzol reagent (Invitrogen, CA, USA) following the manufacturer's instructions. The same volume of the RNA samples $(0.5 \mu \mathrm{g})$ was reverse transcribed into cDNA using a PrimeScript ${ }^{\mathrm{TM}} \mathrm{RT}$ reagent Kit (TaKaRa, Japan). The qPCR was performed in real-time using an SYBR Green qPCR Master Mix (TransGen, Beijing, China) with primers specific for the MELK gene: PMF1 and PMR1. Detection of the housekeeping gene $\beta$-actin was 
Table 1 The primer designed by Primer Premier 5.0 used in this paper

\begin{tabular}{ll}
\hline Primers & Sequence $\left(5^{\prime}\right.$-3') \\
\hline PMF1 & TCACAGAAACAACAGGCAAACA \\
PMR1 & TGGCTTGGAGCAGAAGATAAGTAG \\
$\beta$-actin F1 & GGATGCAGAAGGAGATCACG \\
$\beta$-actin R1 & CTCGTCGTACTCCTGCTTGC \\
& \\
MF1 & GACTAAACTTCCAGGAGG \\
MR1 & TTGGTCTTAGGATAGCC \\
P-MF1 & ATGAATTCATGAAAGATTATGATGAA \\
P-MR1 & TAGGTACCTTAAACCTTGCAGCTAGA \\
\hline
\end{tabular}

performed with the primers $\beta$-actin $\mathrm{F} 1$ and $\beta$-actin $\mathrm{R} 1$, for normalization.

\section{Cloning, plasmid construction, and transfection of MELK}

The whole MELK coding sequence was optimized using the MF1 and MR1 primers designed according to human MELK genome Primer Premier 5.0. Each amplification was detected by $1.5 \%(\mathrm{w} / \mathrm{v})$ agarose gel stained with ethidiumbromide, and the positive amplifications were directly sequenced with primers on an ABI 3370 DNA sequencer at Sangon Company (Shanghai, China). Precise of the date was ensured by sequencing in both directions, and hand assembly using DNAStar software as a sequence editor, the plasmids pEGFP-pMELK and pCMV-HA-pMELK were constructed with primers P-MF1 and P-MR1 (Table 1) designed according to MELK accurate sequence and clone $M E L K$ into the EcoRI and $K p n I$ sites of pEGFP-c1 and pCMV-HA (Clontech Laboratories, CA, USA). All transient transfections were performed using $4 \mu \mathrm{g}$ of recombinant plasmids in 6-well plates of cultured SUVEC cells using Lipofectamine 2000 (Invitrogen, CA, USA), according to the manufacturer's instructions.

\section{Indirect immunofluorescence assay}

Transfected SUVEC cells with the pCMV-HA-pMELK. After $24 \mathrm{~h}$, cells were fixed with $4 \%$ paraformaldehyde for 30 min, permeabilized with $0.2 \%$ Triton X-100/PBS, then incubated with mouse anti-HA tag monoclonal antibody (Sigma-Aldrich), followed by fluorescein isothiocyanate (FITC)-conjugated goat anti-mouse IgG secondary antibody (1:100 dilution; Sigma-Aldrich) for $1 \mathrm{~h}$, and observed under an inverted fluorescence microscope.

\section{Western blot analysis}

Cell samples were lysed in lysis buffer and boiled in SDS sample buffer for $10 \mathrm{~min}$. The proteins in equal amounts of total protein were separated by SDS-polyacrylamide gel electrophoresis followed by transfer onto polyvinylidene fluoride membranes (Millipore, MA, USA). After incubation with primary and secondary antibodies, protein bands were visualized using Luminata Classico Western HRP Substrate (Millipore, MA, USA) according to the manufacturer's instructions.

\section{Co-immunoprecipitation}

Co-immunoprecipitation was performed using a Pierce ${ }^{\circledR}$ Mammalian c-Myc Tag IP/Co-IP Kit (Pierce, IL, USA) according to the manufacturer's instructions. Agarose slurry was washed three times with Tris Buffered Saline plus $0.05 \%$ Tween-20, and immunoprecipitates were released from the agarose slurry by boiling in $25 \mu \mathrm{L}$ non-reducing sample buffer for $5 \mathrm{~min}$. Western blotting was then carried out using the anti-HA tag antibody follow the procedure described above.

\section{Subcellular localization of the protein}

SUVEC cells were washed with PBS $36 \mathrm{~h}$ after transfection and then incubated in growth medium containing $50 \mathrm{nM}$ MitoTracker Red CMXRos (Invitrogen) for mitochondrial labeling at $37{ }^{\circ} \mathrm{C}$ for $30 \mathrm{~min}$. Washed cells with $1 \times$ PBS and incubated with $10 \mu \mathrm{g} / \mathrm{mL}$ Hoechst 33342 (Invitrogen) at $37^{\circ} \mathrm{C}$ for $30 \mathrm{~min}$, and then analyzed using an AIR MP Confocal Microscope (Nikon Instruments, Tokyo, Japan). The results were processed with NIS Viexer software (Nikon Instruments).

\section{Cell apoptosis assay}

SUVEC cells were transfected with specific plasmids, and the medium replaced with new challenge media 


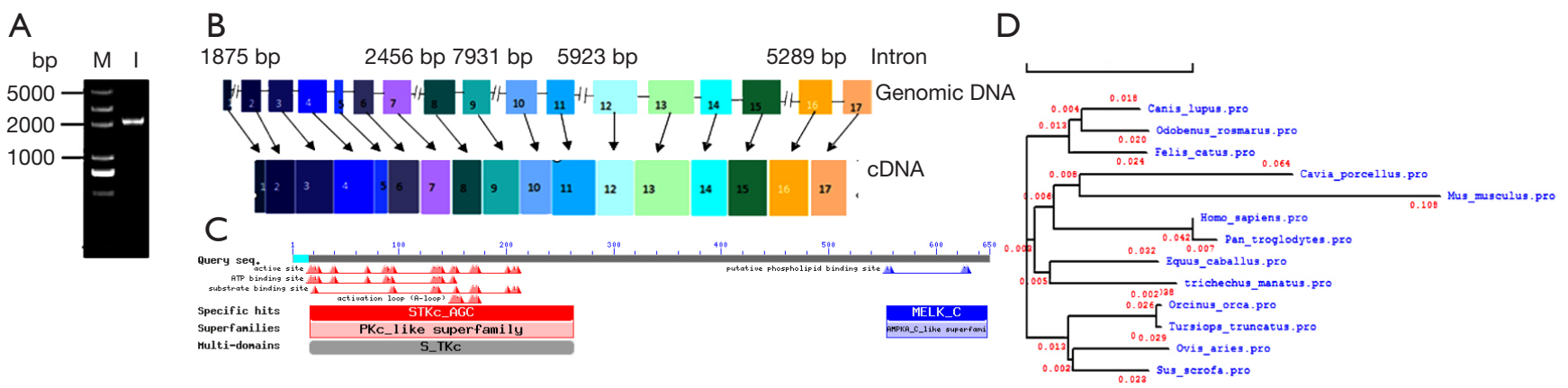

Figure 1 The porcine $M E L K$ gene. (A) Agarose gel of the porcine $M E L K$ cDNA generated by PCR amplification. M, DNA Marker (DL 5000); 1, MELK PCR product. (B) Genomic structure of the porcine MELK gene. (C) Conserved structural domains in the porcine MELK protein predicted by NCBI CDD. (D) Phylogenetic relationship among 11 mammalian species MELK amino acid sequences using DNAMAN.

supplemented with different doses of STS $(0,500$, $1,000 \mathrm{nM})$ (MO, USA). Cells were harvested after $18 \mathrm{~h}$ via detached by trypsin and approximately $5 \times 10^{5}$ cells from each treatment group and then resuspended in working concentration binding buffer (Abcam, MA, USA) and incubated with annexin V-PE/PI (Abcam, MA, USA) for $15 \mathrm{~min}$ at $4{ }^{\circ} \mathrm{C}$. The extent of apoptosis was quantified by flow cytometry (FACS LSRII; Becton Dickinson, San Jose, CA, USA). Dead cells and debris were excluded by selective gating based on electronic cell volume determination. About 20,000 events were collected from each sample.

\section{Statistical analyses}

Calculation of means and standard deviations (SD) and other statistical analyses using SPSS 18.0. Differences between test and control groups were assessed using an independent samples test, with $\mathrm{P}$ values $<0.05$ considered statistically significant and $<0.01$ highly significant.

\section{Results}

\section{Cloning of the full-length pMELK gene}

In this study, the $\mathrm{p} M E L K$ sequence was predicted in silico, and a cDNA containing the whole sequence was obtained for the first time by RT-PCR from the porcine spleen. Using MELK-specific primers PMF1 and PMR1 (Table 1), we amplified a specific band (Figure 1A), sequence analysis found the full length of $\mathrm{p} M E L K$ is $2,072 \mathrm{bp}$ encoding 655 amino acids, which is entirely consistent with Sus scrofa reference sequence (GenBank access No. XM_013993665.1). According to the sites of predicted intron-exon boundaries (GT - AG sequence motif), the sequence contains 17 exons at 50, 88, 118, 147, 71, 96, 101, 72, 101, 87, 130, 125, 235, 101, 170, 107, and $178 \mathrm{bp}$ (Figure $1 B)$. Prediction of the physical and chemical properties of the pMELK protein using the online ExPASy ProtParam tool (http://www.expasy.ch/cgi-bin/protparam) generated a protein molecular formula $\mathrm{C}_{3304} \mathrm{H}_{5280} \mathrm{~N}_{906} \mathrm{O}_{967} \mathrm{~S}_{34}$, with a relative molecular weight of $74.26 \mathrm{KDa}$, theoretical PI value was 8.91 and protein half-life was $30 \mathrm{~h}$, the instability index was computed as 38.69 , indicating that the protein is stable. Using NCBI CDD predicted pMELK protein conserved domains, as shown in Figure 1C, pMELK contains an $\mathrm{S}_{-} \mathrm{TKc}$ protein kinase catalytic domain structure. The phylogenetic relationships among $\mathrm{p} M E L K$ and 10 other mammalian MELK sequences demonstrated MELK is highly evolutionarily conserved, for $>91 \%$ homology and an evolutionary distance $<0.05$ (Table 2, Figure $1 D$ ).

\section{Gene expression of pMELK in different tissues}

A total of 11 tissue samples were isolated from a 30-day-old piglet, including heart, liver, spleen, lung, kidney, tonsil, thymus, superficial inguinal lymph node, hilar lymph node, mesenteric lymph node, and chin lymph node. Quantitative RT-PCR (qRT-PCR) results in triplicate that normalized to the housekeeping gene, $\beta$-actin, showed that porcine $M E L K$ was expressed in all tissues tested; however, there are notable differences among tissues of the expression levels 
Table 2 Comparison of pMELK gene and protein sequences with other mammalian species

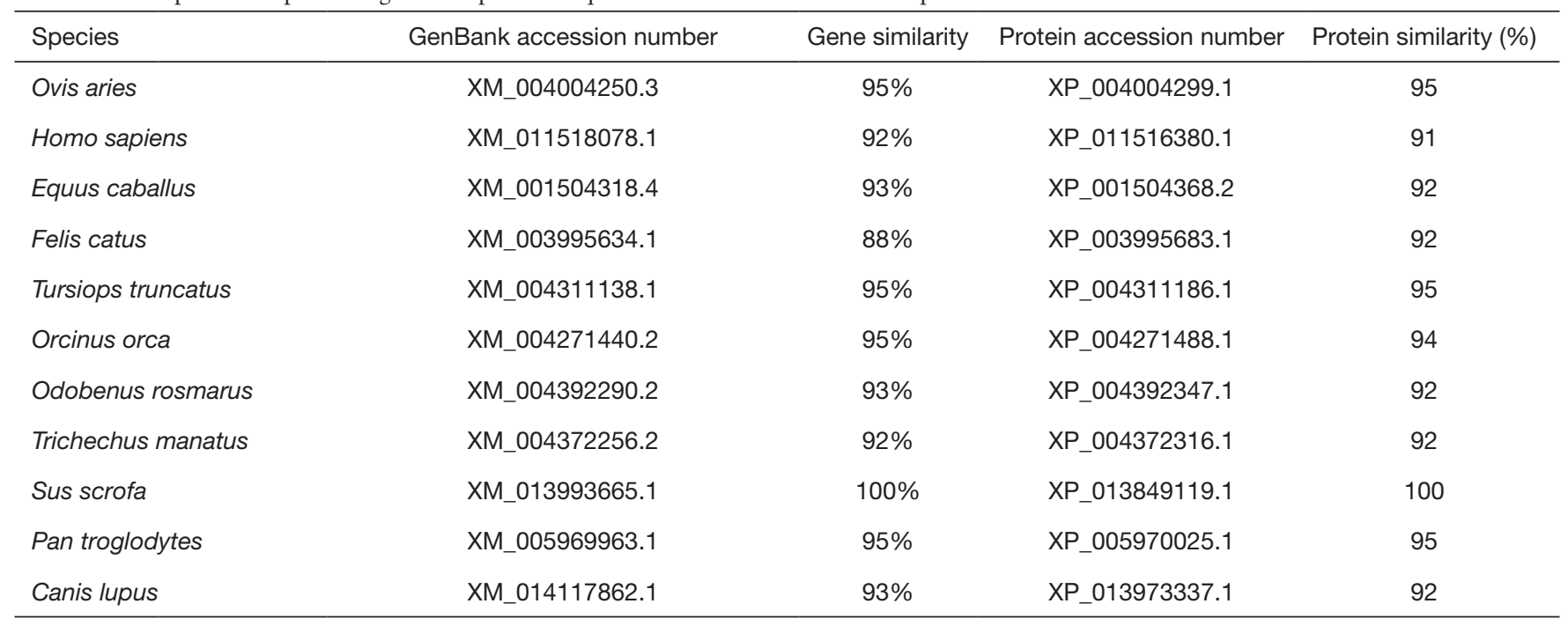

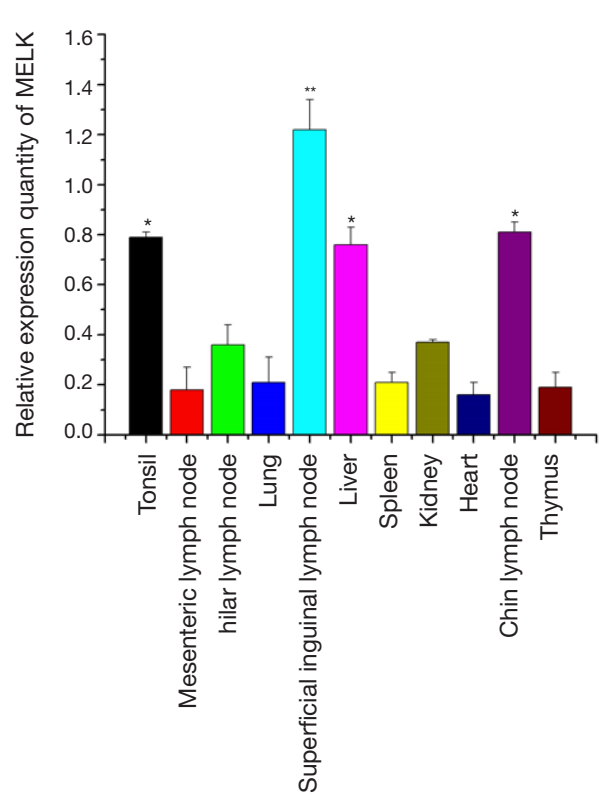

Figure 2 The expression level of MELK mRNA determined by qRT-PCR in different porcine organs.

(Figure 2). The highest expression was in the superficial inguinal lymph node, followed by tonsil, heart, liver, kidney, hilar lymph node, spleen, lung, mesenteric lymph node, and thymus; and the lowest expression was in the chin lymph node. The difference in expression levels in different tissues may relate to their function.
pMELK plasmid construction, expression, and subcellular localization

$\mathrm{p} M E L K$ was cloned into the pEGFP-C1 vector and transformed into Escherichia coli Trans-1 cells (TransGen, Beijing, China); positive clones were selected and verified by sequencing and enzyme digestion, select the clonal that the size, insert location, and the open reading frame was correct (Figure 3A). Green fluorescence in SUVEC cells transfected with pEGFP-C1-MELK and pEGFP-C1 (empty vector) after $24 \mathrm{~h}$ was detected by fluorescence microscopy, whereas no fluorescent signals were detected in negative control cells (Figure 3B). After transfection for $48 \mathrm{~h}$, cells were lysed to detect MELK protein expression by Western blotting. As shown in Figure 3C, a band of approximately $101 \mathrm{kDa}$ was detected from cells transfected with pEGFP-C1-MELK plasmid; this is as expected since EGFP-C1 protein is $27 \mathrm{kDa}$ and MELK protein is about $74 \mathrm{kDa}$. To observe the subcellular localization of MELK protein, the SUVEC cells transfected with pEGFP-C1-MELK plasmid, after $36 \mathrm{~h}$, treat the cells with Hoechst 3,342 dye, under fluorescence microscopy the subcellular localization of MELK was shown to be distributed only in the cytoplasm (Figure 4).

\section{Influence on the morphology of SUVEC cells expressing GFP-pMELK protein treated by STS}

A series of typical apoptotic features were observed by light 

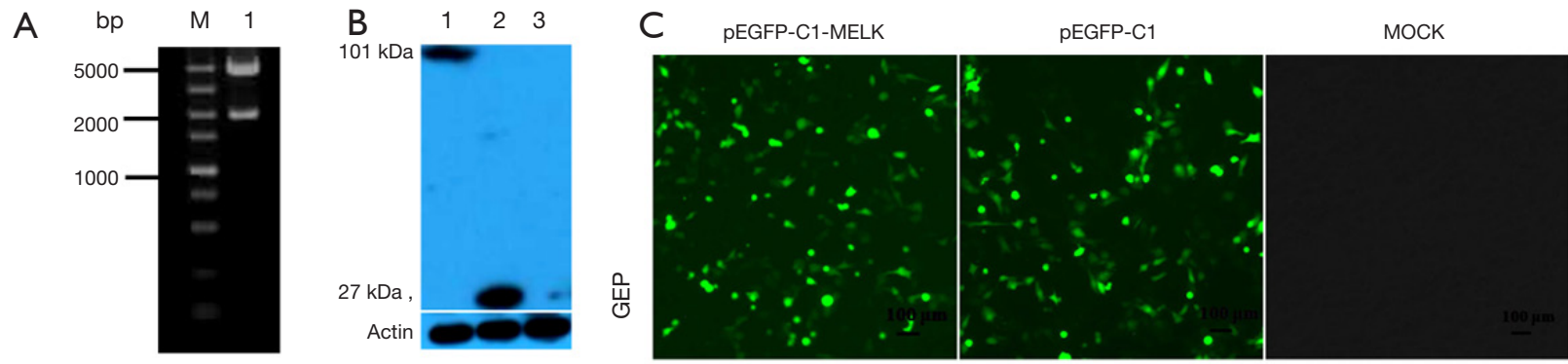

Figure 3 The identification, expression, subcellular localization of pEGFP-C1-MELK (A) Gel electrophoresis of the recombinant pEGFPC1-MELK plasmid digested with EcoRI and KpnI. M, DNA Marker (DL 5000); 1, pEGFP-C1-MELK. (B) Western blotting detected the expression of the pMELK fusion protein in SUVEC cells. 1, SUVEC cells transfected with pEGFP-C1-MELK; 2, SUVEC cells transfected with pEGFP-C1; 3, Untransfected SUVEC cells. (C) Fusion proteins detected by indirect immunofluorescence. 1, SUVEC cells transfected with pEGFP-C1-MELK; 2, SUVEC cells transfected with pEGFP-C1; 3, untransfected SUVEC cells.

microscopy $18 \mathrm{~h}$ after STS treatment of SUVEC cells, which transiently transfected with GFP-pMELK. Such as cells shrank, became spherical, detached, and smaller, deformed, and exhibited a foaming phenomenon in the cell membrane, all of which are typical characteristics of apoptosis, which were not observed in negative control cells (Figure 5). Treatment with Hoechst 33,342 dye of the cells transiently transfected with GFP-pMELK showed typical chromatin condensation, nucleus pyknosis, bright blue fluorescence in the nuclei, which is enhanced when increasing STS concentration. While in negative control cells, cell and nuclear morphology were normal (Figure 5).

\section{pMELK protein enhance STS-induced apoptosis}

STS appropriate treatment dose is $500 \mathrm{nM}$ to SUVEC cells expressing GFP-pMELK protein from above result, so $500 \mathrm{nM}$ STS was added to the SUVEC cells transiently transfected with the GFP-c1 and SUVEC cells transiently transfected with the GFP-pMELK plasmid, under light microscopy, chromatin condensation and nuclear fragmentation were observed, phenomenon is a little serious in SUVEC cells expressing GFPpMELK protein. Then cells were stained with Annexin $\mathrm{V}-\mathrm{PE} / \mathrm{PI}$, and the cell apoptosis rate was analyzed by flow cytometry (Figure $6 A$ ). From the result, cells expressing GFP-pMELK protein showed a little higher rate of apoptosis than the control group expressing GFP protein; statistical analysis of the results in triplicate experiments demonstrated that the pMELK protein could enhance STS-induced apoptosis, but the difference is not significant (Figure 6B).

\section{pMELK interacts with the pBCL-G protein, and the complex is specifically degraded}

Due to the results of bioinformatics analysis and report from human MELK show, pMELK may interact with BCL-G (16), hypothesized that pMELK could also interact with the porcine BCL-G protein (pBCL-G); therefore, we performed western blotting and coimmunoprecipitation experiments to determine whether such an interaction could be detected. We constructed the plasmid HA-pMELK and co-transfected it with a plasmid expressing the myc-pBCL-G protein in SUVEC cells. Both HA-pMELK and myc-pBCL-G protein bands could be detected in cell lysates; however, identification of the products gained by a Pierce ${ }^{\circledR}$ Mammalian c-Myc Tag IP/ Co-IP Kit with anti-HA antibody (to detect HA-pMELK) revealed a band of $30 \mathrm{KDa}$, rather than the expected $78 \mathrm{kDa}$. Also, myc-pBCL-G was not detected in the immunoprecipitate. Moreover, the HA-pMELK protein was not detected in two control immunoprecipitates, either from cells co-expressing myc-pBCL-G and HA-pCMA, or HA-pMELK and myc-pCMA. Given these results, we speculate that $\mathrm{pBCL}-\mathrm{G}$ and $\mathrm{pMELK}$ may interact with one another and that the complex may undergo specific degradation (Figure 7).

\section{pMELK interacts with pBCL-G and affects its subcellular localization}

Since we determined that pMELK can interact with pBCL-G by co-immunoprecipitation, we performed confocal microscopy using Hoechst 33342 and Mito Tracker ${ }^{\circledR}$ Red CMXRos staining to determine whether 


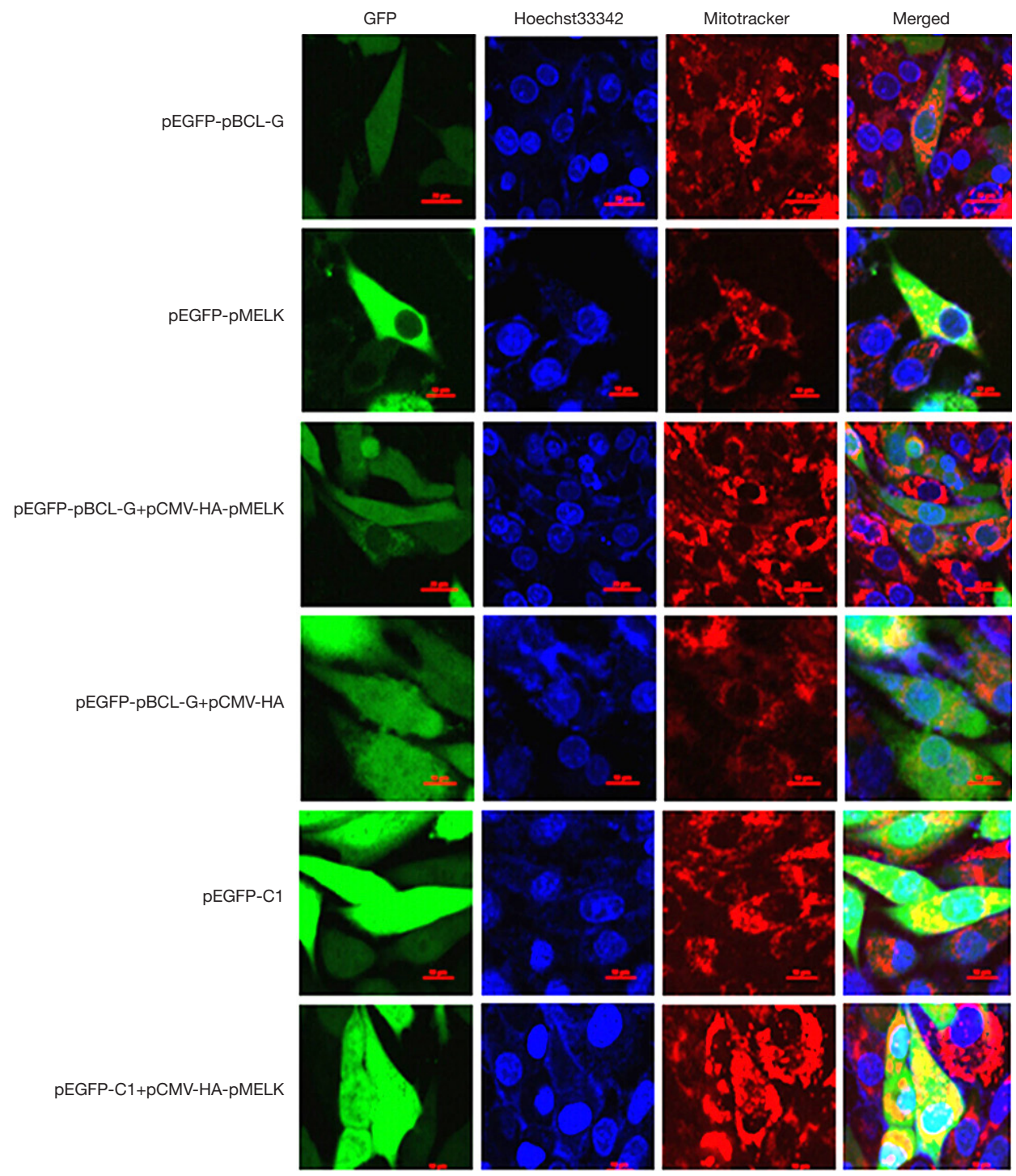

Figure 4 pMELK affects the subcellular localization of the pBCL-G protein. Subcellular localization of GFP-pBCL-G protein was detected by confocal microscopy before and after transfection with pCMV-HA-pMELK or the pCMV-HA empty vector. Cells were stained with Hoechst 33342 and Mito Tracker Red CMXRos. Merged images show the changes in the subcellular localization of the GFP-pBCL-G protein. Scale bar $=10 \mu \mathrm{m}$ in all figures.

pMELK affects the subcellular localization of pBCL-G. As shown in Figure 4, stably expressed GFP-pBCL-G protein was distributed in both nucleus and cytoplasm, whereas when cells were co-transfected with pCMV-HApMELK, GFP-pBCL-G protein was explicitly allocated in the cytoplasm, which was consistent with the subcellular localization of pMELK. Cells transfected with pCMV-HA empty vector, the GFP-pBCL-G protein was distributed both in the nucleus and in the cytoplasm. As a control, SUVEC cells stably expressing GFP protein were also transfected with the pCMV-HA-pMELK vector; the GFP protein was always localized both in the nucleus and cytoplasm.

\section{pMELK significantly suppressed pBCL-G induced apoptosis}

SUVEC cells stably expressing GFP-pBCL-G from our lab were transiently transfected with pCMV-HA-pMELK or pCMV-HA empty vector and after $24 \mathrm{~h}$, treated with $500 \mathrm{nM}$ STS for $18 \mathrm{~h}$, analyzed by flow cytometry to detect 


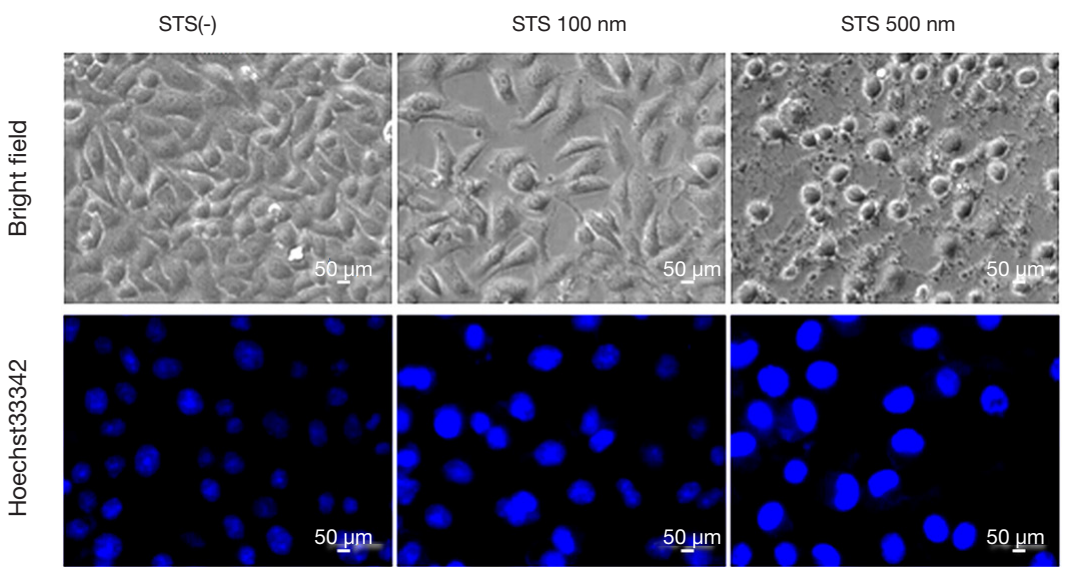

Figure 5 Morphological changes of SUVEC cells over-expressing GFP-pMELK protein treated with 100, 500 nM STS compared with normal SUVEC cells.
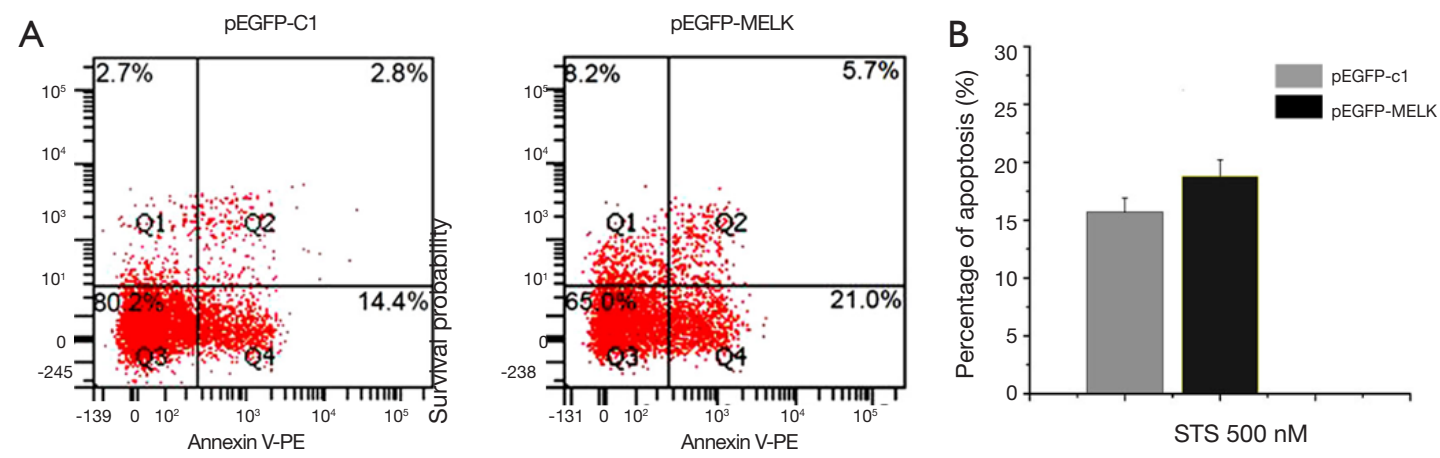

Figure 6 Apoptosis induced by $500 \mathrm{nM}$ STS in SUVEC cells transfected with GFP-pMELK or GFP-c1. (A) Cells were stained with Annexin V-PE/PI and detected by flow cytometry. The lower right quadrant of the scatter diagram is early apoptotic cells, and the upper left quadrant is necrotic cells. (B) Quantitative analysis of Annexin V-positive cells. Data are expressed as means \pm SD, and the experiments were repeated at least three times. There was no statistically significant difference between the two groups $(\mathrm{P}>0.05)$.

apoptosis. As shown in Figure 8A, compared with cells over-expressing pBCL-G alone, added over-expression of pMELK markedly attenuated STS-induced apoptosis of SUVEC cells stably expressing the GFP-pBCL-G protein. These results indicate that pMELK and pBCL-G interacting complex products may have an opposing role to pBCL-G in apoptosis, which is consistent with humans, so the kinase activity of pMELK could be a promising molecular model for the development of therapy for disease caused by BCL-G.

\section{Discussion}

The MELK gene originally was cloned from Mus musculus, yet MELK is one of the stored maternal mRNAs expressed in developing eggs and embryos (20). MELK protein is classified as part of the AMPK/Snf1 family, due to a conserved serine/threonine kinase domain present in its $\mathrm{N}$-terminal region (21). Similar to other family members, the catalytic domain of MELK is adjacent to an UbiquitinAssociated (UBA) domain; however, the kinase activity of MELK is activated by autophosphorylation in vitro, whereas other family members are dependent on phosphorylation by upstream kinases (such as LKB1 or CaMKK2) for their activation, shortly after its discovery, MELK, also known as MPK38, was cloned from a murine teratocarcinoma cell line, PCC4 (22). Mouse MELK expresses itself in the spleen and thymus, but not in the muscle, kidney, or liver. 
MELK expression was also observed in mouse $\mathrm{T}$ lineage lymphocytes and macrophage/monocyte cells but could not be detected in a B cell line so that MELK may have an essential role in signal transduction in specific hematopoietic cell lineages (22). Subsequently, the involvement of MELK in underlying biological mechanisms and functions, including cell signaling pathways and tumor progression, has been determined in the organs and cells of different species (23-28), providing a deeper understanding of the role of this protein. However, until now, there has been no report of porcine MELK or its expression, function, and
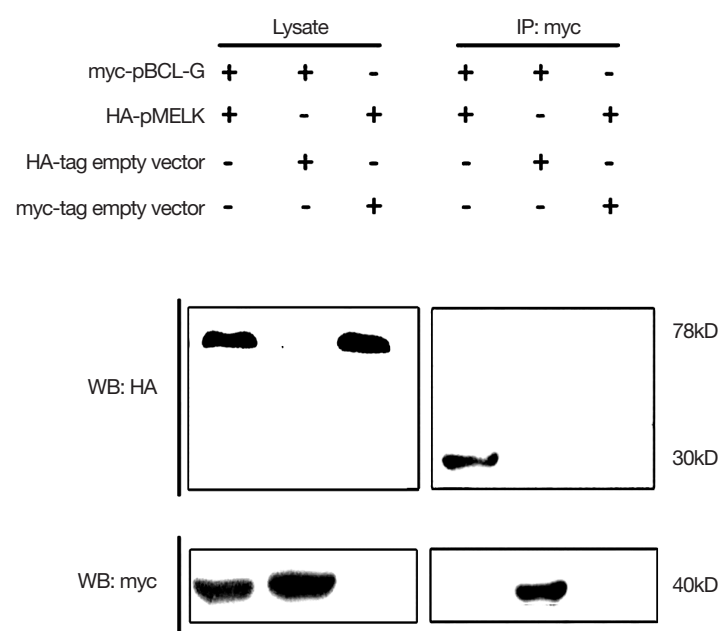

Figure 7 Interaction of pMELK with pBCL-G detected by coimmunoprecipitation. The expression of pMELK and pBCL-G proteins co-transfected in SUVEC cells was examined by western blot analysis. The pMELK protein was degraded from 78 to $30 \mathrm{kDa}$ after interaction with pBCL-G. role in apoptosis.

In this study, we cloned the MELK gene from the spleen of a 30-day-old piglet. The full-length sequence is 2,072 bp with 17 exons encoding 655 amino acids, which is like MELK genes from other species. Phylogenetic analysis showed that MELK is highly evolutionarily conserved among 10 other mammalian species, with an evolutionary distance of $<0.05$. MELK was widely expressed in 11 organ samples isolated from 30-day-old piglets; the highest expression was in the superficial inguinal lymph node and the lowest in the chin lymph node. The observed differential expression levels in various porcine tissues may have functional relevance.

MELK protein structure and function have been studied in various species as model organisms, shedding light on its features in humans. It is conserved throughout species, including the majority of mammalian and nonmammalian species; however, the protein shows slight functional differences among species. Importantly, MELK has been shown to interact with and phosphorylate vital proteins to regulate G2/M cell cycle progression in Xenopus laevis (29). Furthermore, MELK is strongly implicated in cell cycle regulation, proliferation, mitosis, and spliceosome assembly (30). MELK also has a vital role in cell division, cell propagation, and maintenance in organ-specific stem cells from non-mammalian species, whereas, in mammalian systems, MELK is essential for organogenesis, stem cell proliferation, and cell cycle regulation (31). In humans, MELK participates in the development of numerous cancers, including astrocytoma, glioblastoma, breast cancer, and melanoma, through its influence on tumor initiation and propagation. Although many functions of MELK have
A

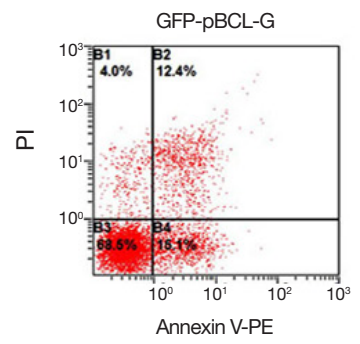

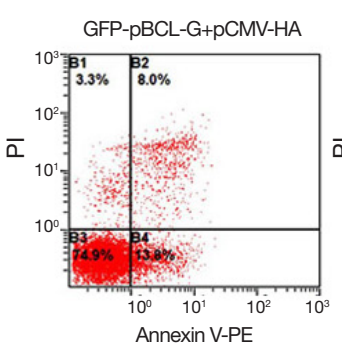

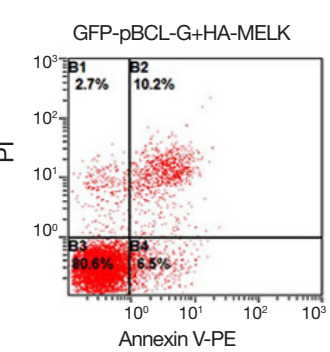

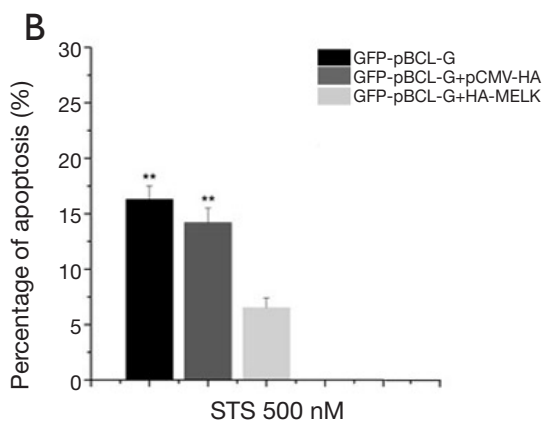

Figure 8 The interaction of pMELK with pBCL-G effects pBCL-G -induced apoptosis. (A) FACS scatter plots representing SUVEC cells treated with STS (500 nM) and co-expressing GFP-pBCL-G alone, GFP-pBCL-G, and pCMV-HA (vector control), or GFP-pBCL-G and pCMV-HA-pMELK. (B) Quantification of Annexin V-positive cells. Data are expressed as means \pm SD, and the experiments were repeated at least three times. ${ }^{* *}$, statistically significant difference $(\mathrm{P}<0.05)$ versus the control group. 
been elucidated, there is little information available about porcine MELK. In our study, we found that pMELK is $74 \mathrm{kDa}$ in size and distributed solely in the cytoplasm. In SUVEC cells, we discovered that pMELK could enhance STS-induced apoptosis; and pMELK did interact with BCL-G, a pro-apoptotic member of the BCL-2 family and affected its subcellular localization. Also, the interaction production between MELK and pBCL-G significantly inhibited STS-induced apoptosis, indicating that the complex production of pMELK interacted with $\mathrm{pBCL}-\mathrm{G}$ plays an opposing role to pBCL-G in apoptosis, similar to findings in humans. Porcine models have advantages for scientific research compared to mice since they are more physiologically and anatomically similar to humans (3234). Therefore, porcine MELK is a potential molecular model for related diseases and inhibition of MELK may be an attractive therapeutic target in several cancers related to humans.

\section{Acknowledgments}

Funding: None.

\section{Footnote}

Conflicts of Interest: The authors have no conflicts of interest to declare.

Ethical Statement: The authors are accountable for all aspects of the work in ensuring that questions related to the accuracy or integrity of any part of the work are appropriately investigated and resolved. All animal experiments were conducted in compliance with current Chinese ethical legislation.

Open Access Statement: This is an Open Access article distributed in accordance with the Creative Commons Attribution-NonCommercial-NoDerivs 4.0 International License (CC BY-NC-ND 4.0), which permits the noncommercial replication and distribution of the article with the strict proviso that no changes or edits are made and the original work is properly cited (including links to both the formal publication through the relevant DOI and the license). See: https://creativecommons.org/licenses/by-nc-nd/4.0/.

\section{References}

1. Gil M, Yang Y, Lee Y, et al. Cloning and expression of a
cDNA encoding a novel protein serine/threonine kinase predominantly expressed in hematopoietic cells. Gene 1997;195:295-301.

2. Janostiak R, Rauniyar N, Lam TT, et al. MELK Promotes Melanoma Growth by Stimulating the NF- B Pathway. Cell Rep 2017;21:2829-41.

3. Cigliano A, Pilo MG, Mela M, et al. Inhibition of MELK Protooncogene as an Innovative Treatment for Intrahepatic Cholangiocarcinoma. Medicina (Kaunas) 2019. doi: 10.3390/medicina56010001.

4. Davezac N, Baldin V, Blot J, et al. Human $\mathrm{pEg} 3$ kinase associates with and phosphorylates CDC25B phosphatase: a potential role for $\mathrm{pEg} 3$ in cell cycle regulation. Oncogene 2002;21:7630-41.

5. Badouel C, Chartrain I, Blot J, et al. Maternal embryonic leucine zipper kinase is stabilized in mitosis by phosphorylation and is partially degraded upon mitotic exit. Exp Cell Res 2010;316:2166-73.

6. Vulsteke V, Beullens M, Boudrez A, et al. Inhibition of spliceosome assembly by the cell cycle-regulated protein kinase MELK and involvement of splicing factor NIPP1.J Biol Chem 2004;279:8642-7.

7. Jiang P, Zhang D. Maternal Embryonic Leucine Zipper Kinase (MELK): A Novel Regulator in Cell Cycle Control, Embryonic Development, and Cancer. Int J Mol Sci 2013;14:21551-60.

8. Marie SK, Okamoto OK, Uno M, et al. Maternal embryonic leucine zipper kinase transcript abundance correlates with malignancy grade in human astrocytomas. Int J Cancer 2008;122:807-15.

9. Kig C, Beullens M, Beke L, et al. Maternal embryonic leucine zipper kinase (MELK) reduces replication stress in glioblastoma cells. J Biol Chem 2013;288:24200-12.

10. Wang R, Chen Y, Yang B, et al. Design, synthesis, biological evaluation and molecular modeling of novel 1H-pyrrolo[2,3-b]pyridine derivatives as potential antitumor agents. Bioorg Chem 2020;94:103474.

11. Brognard J, Hunter T. Protein kinase signaling networks in cancer. Curr Opin Genet Dev 2011;21:4-11.

12. Manning BD. Challenges and opportunities in defining the essential cancer kinome. Sci Signal 2009;2:pe15.

13. Nishitoh H, Saitoh M, Mochida Y, et al. ASK1 is essential for JNK/SAPK activation by TRAF2. Mol Cell 1998;2:389-95.

14. Seong HA; Ha H. Murine protein serine-threonine kinase 38 activates $\mathrm{p} 53$ function through ser 15 phosphorylation. J Biol Chem 2012;287:20797-810.

15. Gu C, Banasavadi-Siddegowda YK, Joshi K, et al. Tumor- 
specific activation of the C-JUN/MELK pathway regulates glioma stem cell growth in a p53-dependent manner. Stem Cells 2013;31:870-81.

16. Lin ML, Park JH, Nishidate T, et al. Involvement of maternal embryonic leucine zipper kinase (MELK) in mammary carcinogenesis through interaction with Bcl-G, a pro-apoptotic member of the Bcl-2 family. Breast Cancer Res 2007; 9:R17.

17. Cordes S, Frank CA, Garriga G. The C. elegans MELK ortholog PIG-1 regulates cell size asymmetry and daughter cell fate in asymmetric neuroblast divisions. Development 2006; 133:2747-56.

18. Denning DP, Hatch V, Horvitz HR. Programmed elimination of cells by caspase-independent cell extrusion in C. elegans. Nature 2012;488:226-30.

19. Hong HX, Zhang YM, Xu H, et al. Immortalization of swine umbilical vein endothelial cells with human telomerase reverse transcriptase. Mol Cells 2007;24:358-63.

20. Cho YS, Yoo J, Park S, et al. The structures of the kinase domain and UBA domain of MPK38 suggest the activation mechanism for kinase activity. Acta Crystallogr D Biol Crystallogr 2014;70:514-21.

21. Pitner MK, Taliaferro JM, Dalby KN, Bartholomeusz C. MELK: a potential novel therapeutic target for TNBC and other aggressive malignancies. Expert Opin Ther Targets 2017;21:849-59.

22. Gil M, Yang Y, Lee Y, et al. Cloning and expression of a cDNA encoding a novel protein serine/threonine kinase predominantly expressed in hematopoietic cells. Gene 1997;195:295-301.

23. Nakano I, Masterman-Smith M, Saigusa K, et al. Maternal embryonic leucine zipper kinase is a key regulator of the proliferation of malignant brain tumors, including brain tumor stem cells. J Neurosci Res 2008;86:48-60.

24. Gray D, Jubb AM, Hogue D, et al. Maternal embryonic leucine zipper kinase/murine protein serine-threonine

Cite this article as: Chen P, Wang J, Wang X, Chen X, Li C, Tan T. Cloning, tissue distribution, expression pattern, and function of porcine maternal embryonic leucine zipper kinase. Ann Transl Med 2020;8(5):239. doi: 10.21037/atm.2020.03.46 kinase 38 is a promising therapeutic target for multiple cancers. Cancer Res 2005;65:9751-61.

25. Wang Y, Lee YM, Baitsch L, et al. MELK is an oncogenic kinase essential for mitotic progression in basal-like breast cancer cells. Elife 2014;3:e01763.

26. Nguyen LV, Vanner R, Dirks P, et al. Cancer stem cells: an evolving concept. Nat Rev Cancer 2012;12:133-43.

27. Hebbard LW, Maurer J, Miller A, et al. Maternal embryonic leucine zipper kinase is upregulated and required in mammary tumor-initiating cells in vivo. Cancer Res 2010;70:8863-73.

28. Joshi K, Banasavadi-Siddegowda Y, Mo X, et al. MELK dependent FOXM1 phosphorylation is essential for proliferation of glioma stem cells. Stem Cells 2013;31:1051-63.

29. Choi HS, Lee SH, Kim H, et al. Germ cell-specific gene 1 targets testis-specific poly(A) polymerase to the endoplasmic reticulum through protein-protein interactions. FEBS Lett 2008;582:1203-9.

30. Chung S, Suzuki H, Miyamoto T, et al. Development of an orally-administrative MELK-targeting inhibitor that suppresses the growth of various types of human cancer. Oncotarget 2012;3:1629-40.

31. Tassan JP. Cortical localization of maternal embryonic leucine zipper kinase (MELK) implicated in cytokinesis in early xenopus embryos. Commun Integr Biol 2011;4:483-5.

32. Codas R, Badet L, Eugene M, Giraud S, et al. Evaluation of pulsatile perfusion machine RM3 for kidney preservation in a swine model of renal autotransplantation. Transplant Proc 2009;41:3296-8.

33. Fodor WL, Williams BL, Matis LA, et al. Expression of a functional human complement inhibitor in a transgenic pig as a model for the prevention of xenogeneic hyperacute organ rejection. Proc Natl Acad Sci USA 1994;91:11153-7.

34. Kozianka J, Kielan W, Waleczek H. Barium peritonitis-a study in pigs. Adv Clin Exp Med 2003;12:569-73. 